\title{
A Shape Constraints Based Method to Recognize Ship Objects from High Spatial Resolution Remote Sensed Imagery
}

\author{
Min Wang, Jiancheng Luo, Chenghu Zhou, and Dongping Ming \\ State Key Laboratory of Resources \& Environmental Information System, \\ Institute of Geographical Sciences and Natural Resources Research, \\ Chinese Academy of Sciences, A11,Datun Road, \\ Anwai, Beijing, 100101, P.R. China \\ wangmalreis.ac.cn
}

\begin{abstract}
Automatically extracting moored ships from high spatial resolution remote sensed imagery is more difficult than offshore ships because their spectrum values and textures are very close to the harbor. For this reason, different routes are designed and applied to extract the two kinds of ships. Our whole method can be divided into three main steps: 1) extract water polygons with histogram segmentation, 2) extract holes in the water polygons with morphological operations as the possible offshore ships, and extract possible moored ship with the identification of the salients to sea along the water boundary, and then 3) screen real ships out of these possible ships with more shape constraints. A case study is carried out on Spot 5 imagery to validate our method.
\end{abstract}

\section{Introduction}

Automatically recognizing and extracting man-made objects from remote sensed imagery has always been an important research task in the fields such as computer vision, pattern recognition and application of remote sensing, etc. Recently, information extraction and object recognition from high spatial resolution remote sensed imagery become one hot spot with the launch of many high resolution spaceborne sensors, such as Ikonos, QuickBird, Spot 5, etc[1][2].

The resolution of high spatial resolution remote sensing can reach less than $10 \mathrm{~m}$, which gives very fine details of the earth surface. It can undertake some tasks in a much cheaper spaceborne remote sensing way which can only be done by aerial remote sensing before, thus has a very wide application scope in photogrammetry, city planning, transportation, military, agriculture, forestry, etc. But, with the improving of resolution, data volume increases remarkably, and spectral distribution become more complicated (e.g., more prevailing substantial spectral confusion), all these bring greater difficulties to information extraction work [3][4].

This paper focuses on automatically extracting one kind of special objects, which are large ships from high resolution remote sensed imagery. To the author's knowledge, most work on ship recognition are on radar imagery (e.g., SAR imagery, 
see literature [5][6][7][8] for examples). In them, for example, Zhou et al. [5] separate sea and land with gray level histogram segmentation, and detect ships in sea with moment invariant threshold from SAR imagery. Eldhuset et al. [6] detect ship targets with moving window filtering on SAR imagery. Besides, Zhang et al. [9] first get edge enhanced image with fuzzy theory, binarize it with segmenting threshold, separate targets and background with filtering operations, and then recognize running ships with combing pixel values and shape features on Spot optical imagery.

We can find that the common ground of most ship target recognition work, which is also adopted in this paper, is firstly to distinguish land and water (if without land in image, this step can be omitted), and then distinguish ships with surrounding water with their pixel values and some shape features. However, the main contributions of our work are:

- We use high spatial resolution optical imagery, a high resolution and good complementarity to radar imagery;

- Our method can recognize moored ships conglutinated to shoreline, which are much difficult to be separated automatically from harbors, for their pixel values and textures are much close between each other.

In this paper, we design different algorithm routes for the recognition and extraction of offshore and moored ships, which combine the shape knowledge of harbor and different running statuses of ships.

The rest of this paper is organized as follows. Section 2 describes our shape constraints based ship recognition method. Section 3 is a case study on Spot 5 imagery. Section 4 sums up our paper.

\section{Shape Constraints Based Method}

In common circumstances, water in high spatial resolution panchromatic imagery has relatively lower pixel values than other objects. For this reason, we can separate offshore ships with surrounding water directly with pixel values. We first extract water with image segmentation, detect those holes in these water areas with mathematics morphology operators (we call those holes "possible ship areas"), and then screen the real ships out from these possible ship areas with more shape constraints.

But for moored ships, different routes should be carried out because simple morphological operations only give very poor results. Our core idea is: because coastline of most harbors is very straight in general, the elliptical or semi-elliptical salients to sea along their coastline are often caused by moored ships. We first detect those parts, and then screen the real ships out from them with more shape constraints.

\subsection{Water Extraction}

The first step of this method is to extract water. For this, we can select histogram segmentation or Markov random field texture classification. We choose histogram segmentation, which automatically get the first valley after the first summit in the gray level histogram as the segmentation threshold $T$. 
The principle of histogram segmentation is:

Regarding the histogram $h(\mathrm{z})$ as a curve, we can then get the histogram valleys with calculating the minima, which satisfy that the first order difference $=0$ and the second order difference $>0$. Then, $T$ can be gotten by [10]:

1. Calculate $h(\mathrm{z})$ of the image $f(x, y)$, and segment it to $N$ levels;

2. Remove burrs with median filtering;

3. Remove possible errors from local dithering with re-smoothing;

4. Calculate the first order difference $h^{\prime}(z)=h(z+1)-h(z)$, and detect from left to right the first position which transits from negative to positive as $T$.

We then segment the image with $T$ with the following rules: water $\leq T$ and nonwater $>T$.

\subsection{Screening Out Ships with Shape Constraints}

We vectorize the water areas and get their boundaries. The work of moored ships extraction would be carried out along their boundaries.

We first set an a priori length range of possible ships $(L)$. It is used to remove those salients whose lengths fall out of $L$ and are impossible to be ships, which can make the following searching and judgment quicker and more precise.

After that, starting from the first node of a water polygon as the begin vertex, we search an end vertex which should satisfies:

- The length of the straight line connecting the two vertices falls in the length range $L$;

- All the other vertices between the two vertices are on the same side of the connecting line, and the area closed by this boundary segment and connecting line protrudes to water.

This area can be regarded as a 'possible ship area'. If this area does exist, then the next searching begins from the end vertex. Otherwise, we move the searching position to the next vertex, and repeat the above searching process until all of the possible ship areas are extracted.

After extracting all these possible offshore and moored ship areas, we screen out the real ships with more shape constraints. We know that real large ships are relatively symmetric between their head and tail, like long and narrow ellipses. But, with many shape influences, which include image precision selection (to accelerate the segmentation of water, we often re-sample the image), outstanding backstays, and conglutination to harbor, the extracted ship areas are often not very regular in shape, and then can not be recognized only by means of 'like a ellipse'.

From several experiments, we define the following shape indices to validate these areas:

1. Simple length index

They are: length range $(L)$ and width range of ship $(W)$.

2. Length to width ratio range $(L W)$

For moored ships, $L W$ is defined as the ratio of the length of the connecting line of the start to end vertices $(l)$ and the max distance of the other vertices to the line $(d)$. The reason to define $L W$ is because the long axes of the enveloping ellipses of moored 
ships are usually parallel to shoreline (see figure 1(a)), and figure 1(b) often represents natural salients. With $L W$, these salients can easily be kicked out from the following searching.

For offshore ships, $L W$ is simply defined as the length ratio of long, short axes.

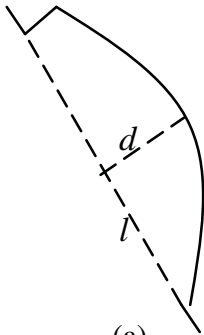

(a)

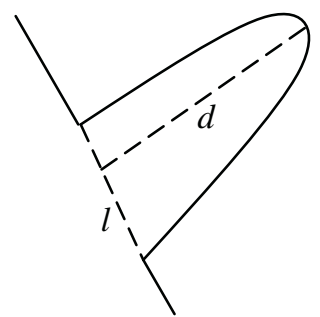

(b)

Fig. 1. Two illustrations of seashore salient

\section{Shape regularity index $(S R)$}

If the possible ship area has much irregular shape (for example, zigzag shape), it should be kicked out. We should find one relatively robust index which can identify irregularity and has the position, size and rotation invariant properties. After many experiments, we choose the third values of the $7 \mathrm{Hu}$ moment invariants [11] as $S R$.

The principle of Hu moments can briefly described as:

Given object boundary curve $C$ and its vertex coordination $f(x, y)$, the $(p+q)$ th order boundary moments are defined as:

$$
m_{p q}=\oint_{C} x^{p} y^{q} d s, p, q=0,1, \ldots .
$$

The central boundary moments are defined as:

$$
\mu_{p q}=\oint_{C}(x-\bar{x})^{p}(y-\bar{y})^{q} d s, p, q=0,1, \ldots,
$$

where

$$
\left\{\begin{array}{l}
\bar{x}=m_{10} / m_{00} \\
\bar{y}=m_{01} / m_{00}
\end{array} .\right.
$$

In discrete cases, for example, digital imagery, we have

$$
\left\{\begin{array}{l}
m_{p q}=\sum_{x, y \in C} x^{p} y^{q} \\
\mu_{p q}=\sum_{(x, y) \in C}(x-\bar{x})^{p}(y-\bar{y})^{q}
\end{array} .\right.
$$


The normalized boundary moments are:

$$
\eta_{p q}=\mu_{p q} / \mu_{00}^{p+q+1} p, q=0,1, \ldots .
$$

The third moment invariant can then be obtained by:

$$
\phi_{3}=\left(\eta_{30}-3 \eta_{12}\right)^{2}+\left(3 \eta_{21}+\eta_{03}\right)^{2} .
$$

From experiments we found that $\phi_{3}$ is often rather small for irregular shapes (e.g., zigzag shape), and it satisfies our need of independent of position, size and orientation, so we use $\phi_{3}$ as $S R$, which can remove all these irregular shapes difficulty to be deleted with other indices.

4. Symmetric regularity index $(S M R)$

$$
S M R=1-\left|\left(A_{0-l / 2}-A_{l / 2-l}\right)\right| / A .
$$

We know that real large ships are commonly near symmetric between head and tail, like narrow ellipses. Considering that possible ship areas are often not very regular in shape, we use area to define symmetry. In formula 7, $A$ is the total area of the possible ship area, $A_{0-1 / 2}$ and $A_{l / 2-l}$ are the partial areas before and after the midpoint of the long axis. $S M R$ can be used to index the symmetry of the possible ship's head and tail.

\section{A Case Study}

Several experiments have been carried out to validate our method. Due to the page limits, here we only give one typical example on Spot 5 image.

The Inputs are:

1. Data source: Spot 5 panchromatic image, with spatial resolution $2.5 \mathrm{~m}$, one harbor in south of China

2. Experiment inputs:

- L: [100-350]meters

- W: [20-60] meters

- LW: [3-10]

- SR: $<=1.0$

- SMR: $>=0.6$

These indices are obtained with visual interpretation and a priori knowledge and as the default inputs.

In this experiment, we get the water threshold with histogram segmentation. From figure 2 we can know that the distribution of pixel values is much concentrated, which makes it difficulty in separating sea and land. What's more, the shape of land, running statuses of ships are much complicated. For example, one ship is not moored directly on the shore, but on a bracket. It can be extracted as offshore ship in our method however.

Figure 3(b) show the water segmentation result with the automatically identified threshold 105. We can see that because the pixel values of some parts of the harbor is very close to water, many small land areas are misclassified into water. To these 
areas, we can use one water area size threshold (for example, 1000 pixels in size) to remove them from the following searching process.

We first extract the offshore ships (in this experiment, the ship on the bracket). With the minimum value of $W 15$, we can determine the temperate size of morphological operator closing, which first dilate then erode the water area. After closing, we then get the possible offshore ships with the result minus the original water area image. From figure 3(c) we can see that many possible offshore ships are conglutinated to shoreline, but most real possible moored ships have not been extracted, which indicates that we can not extract ships simply with morphological operations.

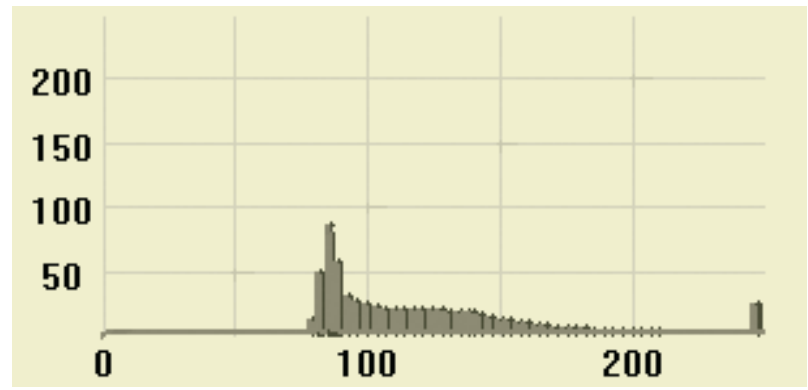

Fig. 2. Gray level histogram of this experiment area

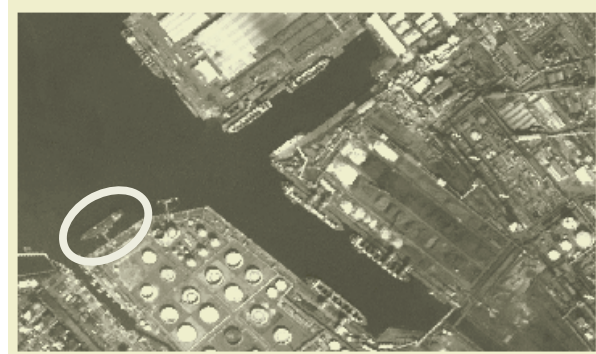

(a)Experiment area

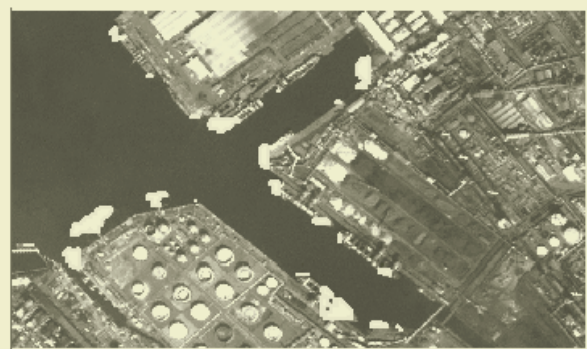

(c)Possible offshore ships

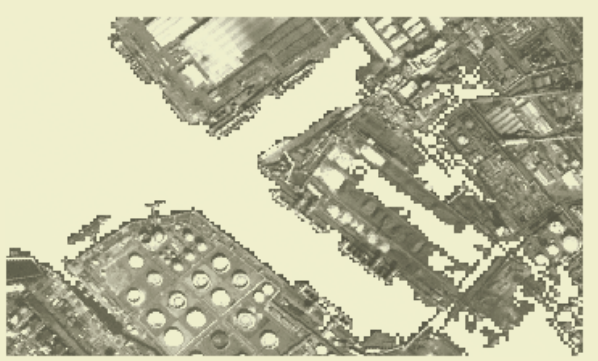

(b)Water

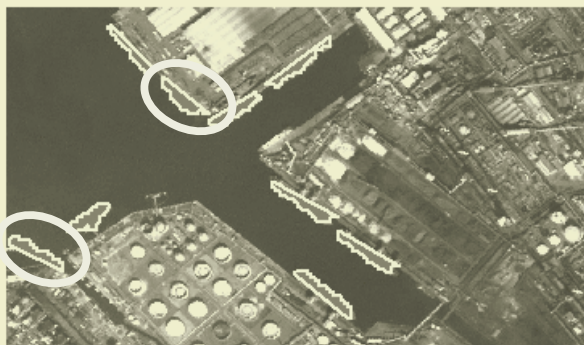

(d)Recognized ships

Fig. 3. A Case Study on Spot 5 imagery 


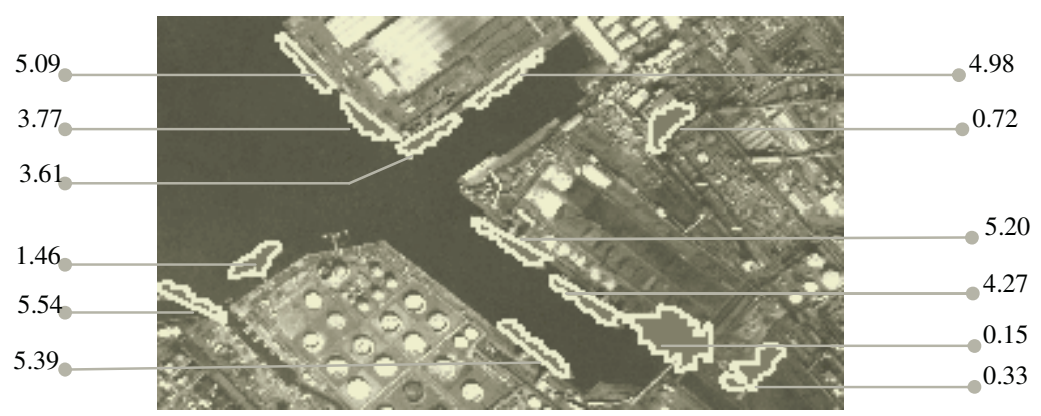

Fig. 4. $S R$ values

We then vectorize the water areas, and extract all these possible moored ships with salient identification. Finally, we extract the real large ships with our shape constraints in all these offshore and moored possible ships.

In this experiment, there exist many irregular possible ship areas, which can not be kicked out with the other shape constraints. From figure 4, it's obvious that the irregular shapes give relatively smaller $S R$ values to the other regular shapes, which indicates $S R$ can be used to denote the regularity of ship areas. With the condition of $S R \leq 1.0$, these irregular shapes can be removed successfully.

The final result is showed in figure 3(d). We can find that all the 7 large ships are extracted, but with 2 misrecognized areas (see the two ellipses). It's reasonable and difficulty to be avoided with our shape constraints based method. For further studies, we can reselect these areas with combining more features of pixel values, textures, which are believed to can further improve the recognizing precision.

From this experiment, we can see that under the complicated distribution of pixel values, harbor shapes and running statuses of the ships, our method get good result however, which indicates that our method is robust and applicable.

\section{Conclusions}

In this paper, a shape constraints based method to recognize ships from high spatial resolution remote sensed imagery is proposed. Because large ships are totally similar in shapes, the inputs of $L W, L R$ and $L M R$ need not be changed frequently, but $L, W$ should be revised a little according to data sources sometimes. Totally speaking, our method is rather simple in use.

In further study, with combing a priori knowledge of 'moored many ships' and shape features of harbors (for example, with relatively straight shoreline and narrow exit), we can try to recognize harbors, the complex man-made objects. We will give detailed discussion in other paper.

Acknowledgment. This work is supported by Chinese National Natural Science Foundation under grant No.40401039, Chinese National Programs for High Technology Research and Development under grand No. 2002AA135230, and Chinese Postdoctoral Foundation. 


\section{References}

[1] Proc. of the processing and application of high spatial resolution remote sensed satellite imagery. Lanzhou, (2003)

[2] Proc. Of IGRASS, Toulouse, France, (2003)

[3] Ma Tin: Technological Model for High Resolution Satellite Images and Information Processing. Information of Remote Sensing, 3(2001)6-10

[4] Cheng Chenqi, Ma Tin: Automatically Extraction of Linear Features from High Resolution Remotely Sensed Imagery. Journal of Remote Sensing,7(2003)26-30

[5] Zou Huanxin, Kuang Gangyao, Jiang Yongmei, Zheng jian: Algorithm of ship targets detection in SAR Ocean Image based on the moment invariant. Computer engineering, 29(2003)114-116

[6] Eldhuset K: An Automatic ship and ship wake detection system for spaceborne SAR images in Coastal Regions. IEEE transactions on GeoScience and Remote Sensing, 34(1996)1010-1018

[7] Q. Jiang, S. Wang, and D. Ziou: Ship Detection in RADARSAT SAR Imagery. In Proc. of CMS, San Diego, (1998)

[8] Qingshan Jiang, Shengrui Wang, Djemel Ziou, and Ali El Zaart: Automatic Detection for Ship Targets in RADARSAT SAR Images from Coastal Regions, In Proc. Of Vision Interface, (1999)131-137

[9] Zhang Yutian, Zeng Anjun: Moving maritime targets detection technology on high spatial resolution remote sensed imagery. Research on telecom technology, 9(2003)1-8

[10] Zhang Yujing: Image segmentation. Beijing: Science press, (2001)

[11] Ming-Kuei Hu: Visual pattern recognition by moment invariants. IRE Transactions on Information Theory, IT-8(1962)179-187 